\title{
Lepton-Quark Collisions at the Large Hadron Collider
}

\author{
Luca Buonocore $\oplus^{1,2,{ }^{*}}$ Ulrich Haisch, ${ }^{3, \dagger}$ Paolo Nason $\odot,{ }^{4, \$}$ Francesco Tramontano, ${ }^{1, \S}$ and Giulia Zanderighi ${ }^{3, \|}$ \\ ${ }^{1}$ Dipartimento di Fisica, Università di Napoli Federico II and INFN, Sezione di Napoli, I-80126 Napoli, Italy \\ ${ }^{2}$ Physik Institut, Universität Zürich, CH-8057 Zürich, Switzerland \\ ${ }^{3}$ Max-Planck-Institut für Physik, Föhringer Ring 6, 80805 München, Germany \\ ${ }^{4}$ Università di Milano-Bicocca and INFN, Sezione di Milano-Bicocca, Piazza della Scienza 3, 20126 Milano, Italy
}

(Received 29 May 2020; revised 29 September 2020; accepted 9 November 2020; published 2 December 2020)

\begin{abstract}
Processes commonly studied at the Large Hadron Collider (LHC) are induced by quarks and gluons inside the protons of the LHC beams. In this Letter, we demonstrate that, since protons also contain leptons, it is possible to target lepton-induced processes at the LHC as well. In particular, by picking a lepton from one beam and a quark from the other beam, we present for the first time a comprehensive analysis of resonant single leptoquark (LQ) production at a hadron collider. In the case of minimal scalar LQs, we derive novel bounds that arise from the LHC Run II considering all possible flavor combinations of an electron or a muon and an up $(u)$, a down $(d)$, a strange, or a charm quark. For the flavor combinations with a $u$ or a $d$ quark, the obtained limits represent the most stringent constraints to date on LQs of this type. The prospects of our method at future LHC runs are also explored. Given the discovery reach of the proposed LQ signature, we argue that dedicated resonance searches in final states featuring a single light lepton and a single light-flavor jet should be added to the exotics search canon of both the ATLAS and the CMS Collaborations.
\end{abstract}

DOI: 10.1103/PhysRevLett.125.231804

Introduction.-At the Large Hadron Collider (LHC) an immense number of collisions between quarks and gluons took place and many more are expected in the upcoming Run III and the high-luminosity (HL LHC) upgrade. These collisions have been studied in great detail and have been used to perform precision measurements of standard model (SM) processes and to search for physics beyond the SM. Because of quantum fluctuations, protons, however, also contain charged leptons, making it possible to study leptoninduced processes at the LHC as well. The simplest process of this kind consists of the collision between a lepton $(\ell)$ from one proton and a quark $(q)$ from the other proton, giving rise to the resonant production of an exotic leptoquark (LQ) state. LQs are hypothetical colored bosons that carry both a baryon number and a lepton number [1]see [2] for a recent LQ review. Below, taking the case of LQs as an example, we demonstrate that lepton-induced processes, which so far have been completely neglected at the LHC, can be complementary to quark- or gluoninduced channels in searches for beyond the SM physics.

A number of different searches for LQs have been considered so far at the LHC. For example, LQs can be pair produced at hadron colliders via quark fusion

Published by the American Physical Society under the terms of the Creative Commons Attribution 4.0 International license. Further distribution of this work must maintain attribution to the author(s) and the published article's title, journal citation, and DOI. Funded by SCOAP ${ }^{3}$. [i.e., $q \bar{q} \rightarrow \mathrm{LQLQ} \rightarrow(\ell q)(\ell q)]$ or gluon fusion [i.e., $g g \rightarrow \mathrm{LQ} \overline{\mathrm{LQ}} \rightarrow(\ell q)(\ell q)]$. For sufficiently large LQlepton-quark couplings, also pair production via $t$-channel exchange of a lepton becomes relevant, and LQ exchange contributes to Drell-Yan like dilepton production $q \bar{q} \rightarrow$ $\ell^{+} \ell^{-}$and single LQ production in $g q \rightarrow \ell^{+} \ell^{-} q$. Both the ATLAS and the CMS Collaborations have exploited the different channels to constrain the parameter space of LQ models (cf. [3-9] for the latest results) and the subject has also received renewed theoretical interest (see, e.g., [10-23]).

In this Letter, we develop a new LQ search strategy, which relies on the fact that once QED interactions are considered, lepton parton distribution functions (PDFs) of the proton $(p)$ are generated. The lepton PDFs are small compared to those of quarks and gluons, since they are suppressed by two powers of the ratio of the electromagnetic coupling constant over the strong coupling constant. A first implementation of a PDF set with leptons was put forward in [24], that was, however, affected by large uncertainties. Based upon the so-called LUX method $[25,26]$, a precise determination of the lepton PDFs has become available recently [27]. In the following, we will use the latter lepton PDF determination to compute the cross sections for resonant single LQ production $\ell q \rightarrow$ LQ $\rightarrow \ell q$ in $p p$ collisions, studying final states with a high transverse momentum $\left(p_{T}\right)$ electron $(e)$ or muon $(\mu)$ and a high- $p_{T}$ light-flavor jet $(j)$, to derive bounds on the parameter space of minimal scalar LQs for all possible flavor combinations involving first- and second-generation 


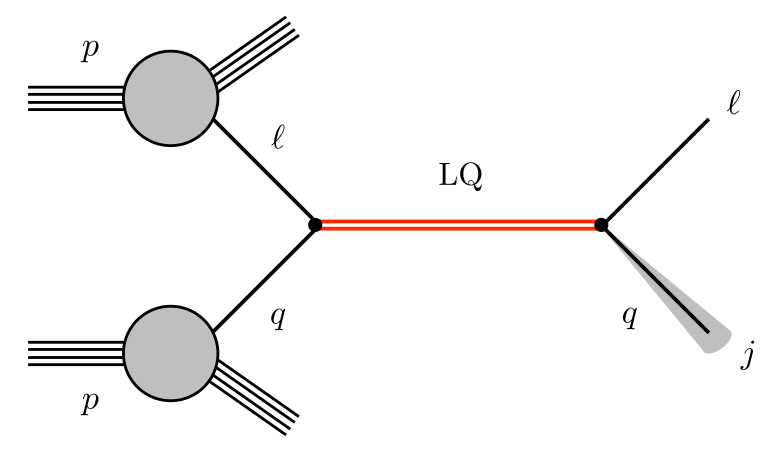

FIG. 1. Feynman diagram describing resonant single LQ production in $p p$ collisions with a final state consisting of a lepton and a jet.

leptons and quarks [28]. The corresponding Feynman diagram is shown in Fig. 1.

$L Q$ interactions.-We follow [18] and consider scalar LQs which couple only to one lepton and quark flavor, taking them to be singlets under the $\mathrm{SU}(2)_{L}$ part of the $\mathrm{SM}$ gauge group. To obtain $\mathrm{SU}(2)_{L}$ invariant interactions, we couple the LQs to the $\mathrm{SU}(2)_{L}$ singlet leptons and quarks, i.e., the right-handed SM fermions. Using the notation where all singlet fields are represented by left-handed charge conjugate fields, the scalar LQ coupling to singlet electrons and up quarks can then be written as

$$
\mathcal{L} \supset \lambda_{e u} \mathrm{LQ}_{e u}\left(E^{c} U^{c}\right)^{*}+\text { H.c., }
$$

where the spinor indices of $E^{c}$ and $U^{c}$ are contracted antisymmetrically. In the limit of large scalar LQ masses, i.e., $M \gg m_{\ell}, m_{q}$, the corresponding total decay width of the LQ is given by

$$
\Gamma=\frac{\left|\lambda_{e u}\right|^{2}}{16 \pi} M
$$

and due to the minimal character of the LQ, it decays almost exclusively to final states with an electron and an up quark. The expressions (1) and (2) also apply to all other flavor combinations after obvious replacements of fields and indices.

Analysis strategy.-The signal predictions corresponding to $s$-channel single LQ production $p p \rightarrow \mathrm{LQ} \rightarrow \ell q$ are generated at leading order (LO) using the implementation of the Lagrangian (1) presented in [14] together with the LUXlep PDF set, which has been obtained by combining the lepton PDFs of [27] with the NNPDF3.1luxQED set [31]. Event generation and showering is performed with MADGRAPH5_aMCNLO [32] and PYTHIA8.2 [33]. Since at present PYTHIA8.2 cannot handle incoming leptonic partons, we have replaced all initial-state leptons by photons in the Les Houches files to shower the events [34]. The parton shower backward evolution of PYTHIA8.2 then produces only quarks from photon splitting, so after showering our simulation includes initial-state quarks instead of leptons [35].

Our analysis uses experimentally identified jets, electrons, muons, and missing transverse energy $\left(E_{T, \text { miss }}\right)$. FastJet3 [37] is used to construct anti- $k_{t}$ jets [38] of radius $R=0.4$. Our analysis is implemented in CheckMATE2 [39], which employs DELPHES3 [40] as a fast detector simulator. Detector effects are simulated by smearing the momenta of the reconstructed objects, and by applying reconstruction and identification efficiency factors tuned to mimic the performance of the ATLAS detector. In particular, electron candidates are required to satisfy the tight identification criteria of ATLAS [41], while muon candidates must fulfil the ATLAS quality selection criteria optimized for high- $p_{T}$ performance $[42,43]$. The corresponding reconstruction and identification efficiency for electrons amounts to $90 \%$ for $p_{T}>500 \mathrm{GeV}$, while for muons the reconstruction and identification efficiency is $69 \%(57 \%)$ at $p_{T}=1 \mathrm{TeV}$ $\left(p_{T}=2.5 \mathrm{TeV}\right)$ —cf. for example [44,45]. $E_{T \text {,miss }}$ is reconstructed from the sum of the smeared calorimeter deposits, including an extra smearing factor that effectively parametrizes additional QCD activity due to pileup and has been tuned to match the ATLAS distributions.

The basic selections in our signal region require a lepton $\left(e\right.$ or $\mu$ ) with $\left|\eta_{\ell}\right|<2.5$ and $p_{T, \ell}>500 \mathrm{GeV}$ and a light-flavor jet with $\left|\eta_{j}\right|<2.5$ and $p_{T, j}>500 \mathrm{GeV}$. We furthermore demand $E_{T \text {,miss }}<50 \mathrm{GeV}$, veto events that contain additional leptons with $\left|\eta_{\ell}\right|<2.5$ and $p_{T, \ell}>7 \mathrm{GeV}$ and impose a jet veto on subleading jets with $\left|\eta_{j}\right|<2.5$ and $p_{T, j}>30 \mathrm{GeV}$. The jet veto limits the amount of hadronic activity and ensures that the background from $t \bar{t}$, and $s$ - and $t$-channel single top production are negligible in the signal region.

The dominant background turns out to be $W^{-}+j$ production which is generated at next-to-leading order (NLO) in QCD. Next-to-next-leading order QCD and electroweak effects that would effectively reduce the size of the $W^{-}+j$ background prediction in the phase space region of interest [46] are not included in our analysis. Subleading backgrounds arise from $Z+j, W W, W^{-} Z$, and $t W$ production and are simulated at $\mathrm{LO}$ and normalized to the known NLO QCD cross sections. At high values of $m_{\ell j}$ also $\ell^{-}+j$ production from an initial-state lepton and quark via $t$-channel exchange of a photon or $Z$ boson represents a relevant irreducible background. We include this background at LO. For each background process, the number of events after cuts is fitted and extrapolated to high invariant lepton-jet masses $m_{\ell j}$ using $e^{-a} m_{\ell j}^{b+c \ln m_{\ell j}}$. This functional form is commonly used in experimental searches (see, e.g., [45]).

In actual experimental analyses the background contributions from events with fake or nonprompt electrons from the decay of heavy-flavor hadrons is typically extracted from the data using a matrix method as described, for 


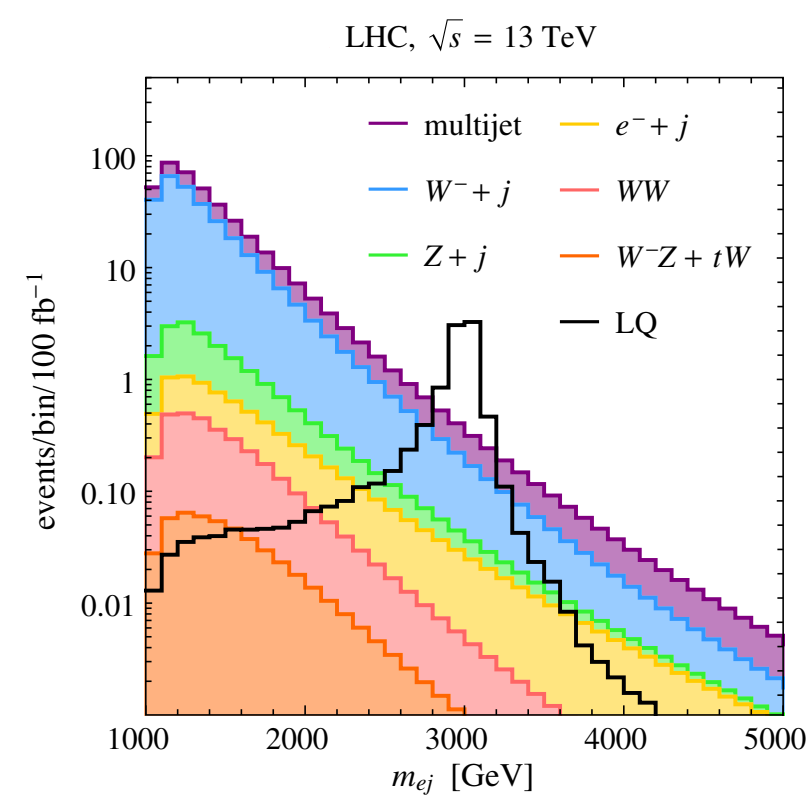

FIG. 2. Distributions of $m_{e j}$ after imposing the experimental selection requirements as detailed in the text. The colored histograms are stacked and represent the SM backgrounds. The LQ signal prediction corresponds to $M=3 \mathrm{TeV}$ and $\lambda_{e u}=1$ and is superimposed as a black line. The events are binned in $100 \mathrm{GeV}$ bins and all predictions are obtained for $100 \mathrm{fb}^{-1}$ of $p p$ collisions at a center-of-mass energy of $\sqrt{s}=13 \mathrm{TeV}$.

example, in [47]. Since we cannot perform such a datadriven background estimate, we mimic the impact of the multijet background in our analysis by taking it from the ATLAS $\ell+j$ analysis [48]. Using the auxiliary material of [48] as provided in [49] we estimate that the ratio of the multijet to the other $e^{-}+j$ backgrounds amounts to $28 \%$, $84 \%$, and $142 \%$ for $m_{e j}$ values of $1 \mathrm{TeV}, 3 \mathrm{TeV}$, and $5 \mathrm{TeV}$, respectively.

In Fig. 2 the distributions of the invariant mass $m_{e j}$ of the electron and the leading jet are displayed for the SM backgrounds, and for a benchmark minimal scalar LQ with mass $M=3 \mathrm{TeV}$ and $\lambda_{e u}=1$, after applying the event selection described above. An integrated luminosity of $100 \mathrm{fb}^{-1}$ under LHC Run II conditions is assumed. Our benchmark LQ has a width of $\Gamma \simeq 60 \mathrm{GeV}$. One observes that the sum of the SM backgrounds is a steeply falling distribution, while the LQ signal exhibits a narrow peak as indicated by the black line. The $t \bar{t}$, and $s$ - and $t$-channel single top backgrounds are not shown, since they are very small.

Systematic uncertainties are treated as follows in our analysis. The scale (PDF) uncertainties affecting the dominant $W^{-}+j$ background have been determined with MADGRAPH5_aMCNLO. They amount to $4.6 \%(1.2 \%), 12 \%$ (4.2\%), and $41 \%$ (57\%) for $m_{\ell j}$ values of $1 \mathrm{TeV}, 3 \mathrm{TeV}$, and $5 \mathrm{TeV}$, respectively. No systematic uncertainty is applied to the signal predictions [50]. The individual sources of uncertainty are added in quadrature which results in total systematic background uncertainties of $4.7 \%, 13 \%$, and $70 \%$ for $m_{\ell j}$ values of $1 \mathrm{TeV}, 3 \mathrm{TeV}$, and $5 \mathrm{TeV}$, respectively.

LHC constraints.-The resonance line shape of the LQ signal is modeled by a relativistic Breit-Wigner that is fitted to the distribution of events after showering, reconstruction, and cuts. In this way the broadening of the peak by PDF, parton shower, and nonperturbative effects is described. We find that compared to (2) the peak is broadened by the latter effects by a factor of 6.0, 3.1, and 2.2 for a LQ mass of $1 \mathrm{TeV}, 3 \mathrm{TeV}$, and $5 \mathrm{TeV}$, respectively.

The statistical significance of any localized excess in the $m_{\ell j}$ distribution is quantified using a sliding window approach after binning the background and signal predictions. The bin size is thereby taken to be equal to the $m_{\ell j}$ resolution. This resolution is estimated by combining the information on the dilepton and dijet mass resolutions given in [44,51] and [52,53], respectively. Using a simple error propagation, we find that in the electron case the experimental mass resolution amounts to $2.3 \%, 1.7 \%$, and $1.6 \%$ at $1 \mathrm{TeV}, 3 \mathrm{TeV}$, and $5 \mathrm{TeV}$, respectively. In the muon case the corresponding numbers are $6.7 \%, 12 \%$, and $17 \%$. The width of the search window is then varied from a minimum of twice the $m_{\ell j}$ resolution up to $2 \mathrm{TeV}$, and the optimal width is determined for each signal hypothesis such that the LQ signal deviates most significantly from the smooth background distribution. The significance is calculated as a Poisson ratio of likelihoods modified to incorporate systematic uncertainties on the background using the Asimov approximation [54].

Figure 3 displays the most relevant $95 \%$ confidence level (C.L.) limits on the magnitude of the couplings $\lambda_{e q}$ as a function of the mass $M$ for minimal scalar LQs. All possible flavor combinations involving an electron and an up $(u)$, a down $(d)$, a strange $(s)$, or a charm $(c)$ quark are considered. The bounds that derive from our novel search strategy for resonant single LQ production are displayed as red (LHC Run II constraints) and orange (projections) shaded regions. The dash-dotted red, solid red, and dashed orange lines assume an integrated luminosity of 36, 139, and $300 \mathrm{fb}^{-1}$ for $p p$ collisions at $\sqrt{s}=13 \mathrm{TeV}$, respectively, while the dotted orange lines assume $3 \mathrm{ab}^{-1}$ of $\sqrt{s}=14 \mathrm{TeV}$ data. The most stringent limits on the mass of minimal first-generation scalar LQs [9] obtained from pair-production (PP) searches, are indicated as black lines. These limits are based on $36 \mathrm{fb}^{-1}$ of LHC Run II data and correspond to $M>1435 \mathrm{GeV}$ for first-generation LQs. Notice that PP via $t$-channel exchange of a lepton has not been considered in the CMS analyses. As shown in [18] this simplification has, however, a minor impact for $\left|\lambda_{e q}\right| \lesssim 1$. Following [18] we add the lepton exchange contribution and indicate the PP bounds by dotted black lines for $\left|\lambda_{e q}\right|>1$. The green lines correspond to the DrellYan (DY) bounds derived in [18] from the CMS results [55], while the yellow lines depict the single production projections [18] of the CMS LHC Run I search [56]. 

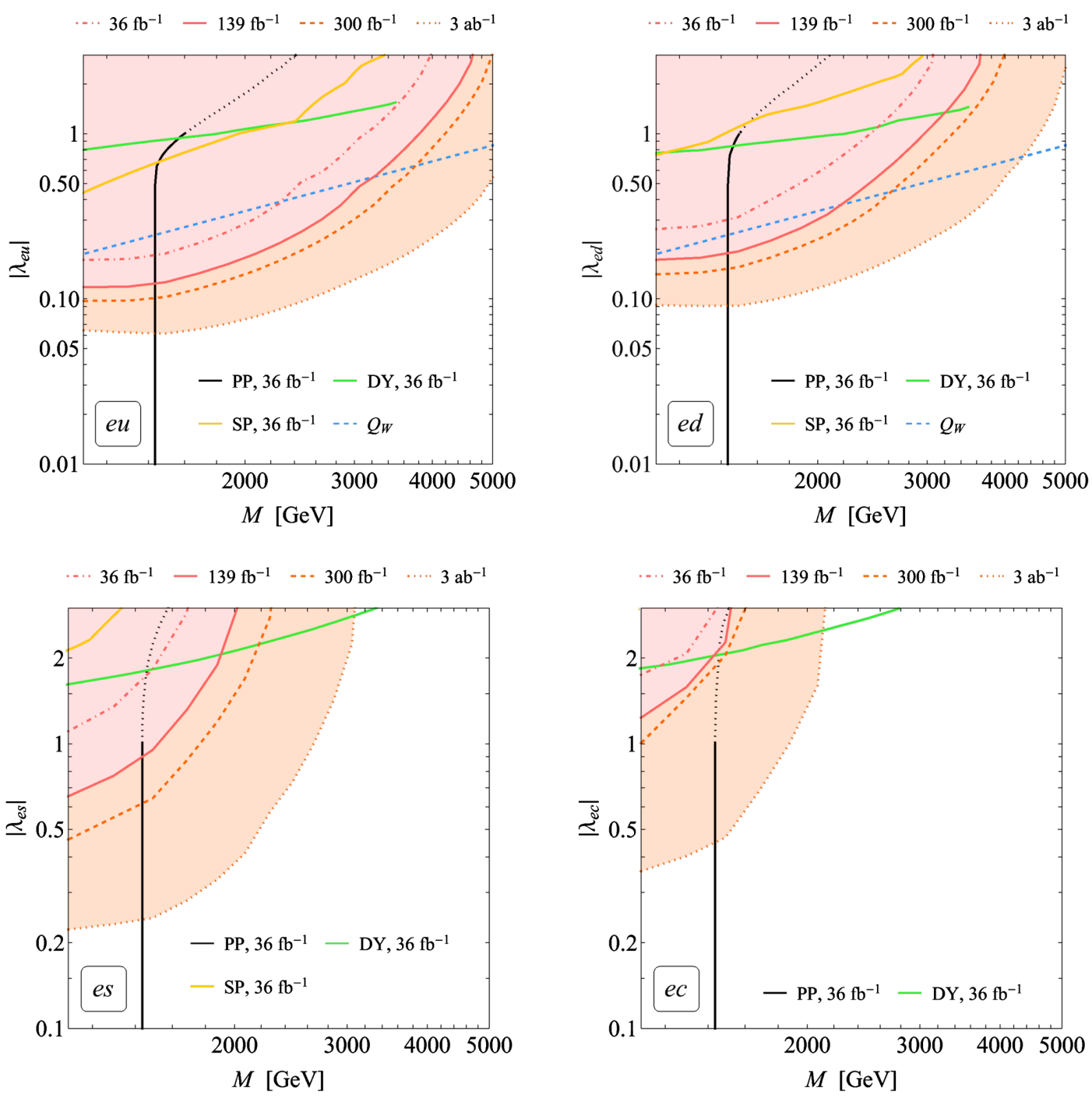

FIG. 3. $95 \%$ C.L. limits on the parameter space of minimal $\mathrm{LQ}_{e q}$ bosons with $q=u, d, s, c$. The red (orange) shaded regions correspond to the parameter space that is excluded by resonant single LQ production at the LHC Run II (future LHC runs). The black lines indicate the PP limits obtained in [18] by recasting the results [9], the green lines correspond to the DY bounds derived in [18] using [55], while the yellow lines represent the single production projections [18] of the search [56]. The dashed blue lines depict the constraints from $Q_{W}$ measurements [18]. The parameter spaces to the left and/or above the lines are ruled out.

Both sets of bounds assume $36 \mathrm{fb}^{-1}$ of $p p$ collisions at $\sqrt{s}=13 \mathrm{TeV}$. The couplings $\lambda_{\text {eq }}$ with $q=u, d$ are also subject to the constraints arising from atomic parity violation, and from parity-violating electron scattering experiments that measure the weak charge $\left(Q_{W}\right)$ of protons and nuclei. The relevant 95\% C.L. bound reads $\left|\lambda_{e q}\right|<$ $0.17 \mathrm{M} / \mathrm{TeV}$ [18] and is shown in the upper two panels of Fig. 3 as a dashed blue line. One sees that for $\lambda_{e u}\left(\lambda_{e d}\right)$ the hypothetical $139 \mathrm{fb}^{-1}$ bounds obtained from $s$-channel single LQ production are more stringent than the constraints from $Q_{W}$ measurements for $M \lesssim 3.2 \mathrm{TeV}$ $(M \lesssim 2.1 \mathrm{TeV})$. At the end of the HL LHC the corresponding limits can be expected to surpass the bounds from $Q_{W}$ measurements for minimal scalar LQ masses up to around 5.7 TeV (4.2 TeV). Strong bounds on the couplings $\lambda_{\mu u}$ and $\lambda_{\mu d}$ are also obtained using our method (cf. [57]), while the minimal scalar LQ interactions proportional to $\lambda_{\ell s}$ or $\lambda_{\ell c}$ are more difficult to constrain given the suppression of the $s$ - and $c$-quark PDFs.

While we have focused in our Letter on limits for the minimal scalar LQs in $\ell^{-}+j$ final states predicted by (1), we expect searches for nonminimal scalar LQs decaying to $\ell^{+}+j$ to give practically identical constraints. Imposing the same selection criteria on the $W^{+}+j$ background process leads to a similar size and shape as for the $W^{-}+j$ process, since the larger $W^{+}+j$ cross section is compensated by a lower $p_{T}$ of the charged lepton in the $W^{+} \rightarrow$ $\ell^{+} \nu_{\ell}$ decay.

In view of the simplicity of the proposed LQ signature and its discovery reach, we urge the ATLAS and CMS Collaborations to add resonance searches in final states 
featuring a single light lepton and a single light-flavor jet to their exotics search canon.

U. H. thanks Jonas Lindert and Giacomo Polesello for helpful discussions. We thank Johannes Blümlein for making us aware of [29]. The research of L. B. is supported in part by the Swiss National Foundation under Contracts No. 200020_188464 and No. IZSAZ2_173357. P. N. acknowledges support from Fondazione Cariplo and Regione Lombardia, Grant No. 2017-2070, and from INFN.

*luca.buonocore@na.infn.it

thaisch@mpp.mpg.de

*paolo.nason@mib.infn.it

§francesco.tramontano@cern.ch

"zanderi@mpp.mpg.de

[1] J. C. Pati and A. Salam, Phys. Rev. D 10, 275 (1974); 11, 703(E) (1975).

[2] I. Doršner, S. Fajfer, A. Greljo, J. F. Kamenik, and N. Košnik, Phys. Rep. 641, 1 (2016).

[3] M. Aaboud et al. (ATLAS Collaboration), New J. Phys. 18, 093016 (2016).

[4] M. Aaboud et al. (ATLAS Collaboration), Eur. Phys. J. C 79, 733 (2019).

[5] M. Aaboud et al. (ATLAS Collaboration), J. High Energy Phys. 06 (2019) 144.

[6] V. Khachatryan et al. (CMS Collaboration), J. High Energy Phys. 03 (2017) 077.

[7] A. M. Sirunyan et al. (CMS Collaboration), Phys. Rev. D 99, 032014 (2019).

[8] A. M. Sirunyan et al. (CMS Collaboration), J. High Energy Phys. 03 (2019) 170.

[9] A. M. Sirunyan et al. (CMS Collaboration), Phys. Rev. D 99, 052002 (2019).

[10] T. Mandal, S. Mitra, and S. Seth, J. High Energy Phys. 07 (2015) 028.

[11] D. A. Faroughy, A. Greljo, and J. F. Kamenik, Phys. Lett. B 764, 126 (2017).

[12] N. Raj, Phys. Rev. D 95, 015011 (2017).

[13] B. Diaz, M. Schmaltz, and Y.-M. Zhong, J. High Energy Phys. 10 (2017) 097.

[14] I. Doršner and A. Greljo, J. High Energy Phys. 05 (2018) 126.

[15] G. Hiller, D. Loose, and I. Nišandžić, Phys. Rev. D 97, 075004 (2018).

[16] S. Bansal, R. M. Capdevilla, A. Delgado, C. Kolda, A. Martin, and N. Raj, Phys. Rev. D 98, 015037 (2018).

[17] A. Angelescu, D. Bečirević, D. A. Faroughy, and O. Sumensari, J. High Energy Phys. 10 (2018) 183.

[18] M. Schmaltz and Y.-M. Zhong, J. High Energy Phys. 01 (2019) 132.

[19] T. Mandal, S. Mitra, and S. Raz, Phys. Rev. D 99, 055028 (2019).

[20] M. J. Baker, J. Fuentes-Martín, G. Isidori, and M. König, Eur. Phys. J. C 79, 334 (2019).

[21] K. Chandak, T. Mandal, and S. Mitra, Phys. Rev. D 100, 075019 (2019).

[22] R. Padhan, S. Mandal, M. Mitra, and N. Sinha, Phys. Rev. D 101, 075037 (2020).
[23] A. Bhaskar, T. Mandal, and S. Mitra, Phys. Rev. D 101, 115015 (2020).

[24] V. Bertone, S. Carrazza, D. Pagani, and M. Zaro, J. High Energy Phys. 11 (2015) 194.

[25] A. Manohar, P. Nason, G. P. Salam, and G. Zanderighi, Phys. Rev. Lett. 117, 242002 (2016).

[26] A. V. Manohar, P. Nason, G. P. Salam, and G. Zanderighi, J. High Energy Phys. 12 (2017) 046.

[27] L. Buonocore, P. Nason, F. Tramontano, and G. Zanderighi, J. High Energy Phys. 08 (2020) 019.

[28] The same idea has already been submitted two decades ago $[29,30]$, but a reliable estimate of the lepton PDFs was missing, and the resulting LHC phenomenology has not been studied in detail.

[29] J. Ohnemus, S. Rudaz, T. Walsh, and P. Zerwas, Phys. Lett. B 334, 203 (1994).

[30] O. J. P. Eboli, R. Z. Funchal, and T. L. Lungov, Phys. Rev. D 57, 1715 (1998).

[31] V. Bertone, S. Carrazza, N. P. Hartland, and J. Rojo (NNPDF Collaboration), SciPost Phys. 5, 008 (2018).

[32] J. Alwall, R. Frederix, S. Frixione, V. Hirschi, F. Maltoni, O. Mattelaer, H. S. Shao, T. Stelzer, P. Torrielli, and M. Zaro, J. High Energy Phys. 07 (2014) 079.

[33] T. Sjöstrand, S. Ask, J. R. Christiansen, R. Corke, N. Desai, P. Ilten, S. Mrenna, S. Prestel, C. O. Rasmussen, and P.Z. Skands, Comput. Phys. Commun. 191, 159 (2015).

[34] This replacement leads to a mismodeling of the signal strength after imposing the lepton and jet veto present in our analysis. We estimate this effect to be of $\mathcal{O}(10 \%)$, and therefore to only mildly affect the derived LQ limits.

[35] We remark that the inclusion of incoming leptons in shower Monte Carlos is not difficult to realize and one can expect it to become available in the near future [36].

[36] P. Richardson and T. Sjöstrand (private communication).

[37] M. Cacciari, G. P. Salam, and G. Soyez, Eur. Phys. J. C 72, 1896 (2012).

[38] M. Cacciari, G. P. Salam, and G. Soyez, J. High Energy Phys. 04 (2008) 063.

[39] D. Dercks, N. Desai, J. S. Kim, K. Rolbiecki, J. Tattersall, and T. Weber, Comput. Phys. Commun. 221, 383 (2017).

[40] J. de Favereau et al. (DELPHES 3 Collaboration), J. High Energy Phys. 02 (2014) 057.

[41] G. Aad et al. (ATLAS Collaboration), J. Instrum. 14, P12006 (2019).

[42] G. Aad et al. (ATLAS Collaboration), Eur. Phys. J. C 76, 292 (2016).

[43] G. Aad et al. (ATLAS Collaboration), J. Instrum. 15, P09015 (2020).

[44] G. Aad et al. (ATLAS Collaboration), Phys. Lett. B 796, 68 (2019).

[45] G. Aad et al. (ATLAS Collaboration), Phys. Rev. D 100, 052013 (2019).

[46] J. Lindert et al., Eur. Phys. J. C 77, 829 (2017).

[47] M. Aaboud et al. (ATLAS Collaboration), Phys. Lett. B 762, 334 (2016).

[48] G. Aad et al. (ATLAS Collaboration), Phys. Rev. Lett. 112, 091804 (2014).

[49] G. Aad et al. (ATLAS Collaboration), Report No. CERNPH-EP-2013-193 (2013). 
[50] The scale (PDF) uncertainties of the signal amount to less than $10 \%(3 \%)$ for LQ masses between $1 \mathrm{TeV}$ and $5 \mathrm{TeV}$. These uncertainties would hence affect the limits on $\lambda_{\ell q}$ at the few percent level only.

[51] G. Aad et al. (ATLAS Collaboration), hepdata.88425.v1 (2019), https://www.hepdata.net/record/ins1725190?version=1.

[52] G. Aad et al. (ATLAS Collaboration), J. High Energy Phys. 03 (2020) 145.

[53] G. Aad et al. (ATLAS Collaboration), hepdata.91126.v1 (2019), https://www.hepdata.net/record/ins1759712? version=1.
[54] G. Cowan, http://www.pp.rhul.ac.uk/ cowan/stat/medsig/ medsigNote.pdf (2012).

[55] A. M. Sirunyan et al. (CMS Collaboration), J. High Energy Phys. 06 (2018) 120.

[56] V. Khachatryan et al. (CMS Collaboration), Phys. Rev. D 93, 032005 (2016); 95, 039906(E) (2017).

[57] See the Supplemental Material at http://link.aps.org/ supplemental/10.1103/PhysRevLett.125.231804 for a summary of the $95 \%$ C.L. limits on the magnitude of the couplings $\lambda_{\mu q}$. 